\title{
La planeación de los escritos a partir del propósito comunicativo, mensaje y destinatario en estudiantes de grado 3 del colegio Juan XXIII de Macaravita ${ }^{1}$
}

\begin{abstract}
The planning of writings from the communicative purpose, message and recipient in students of grade 3 of the college Juan XXIII of Macaravita
\end{abstract}

\begin{abstract}
O planejamento dos escritos a partir do propósito comunicativo, mensagem e destinatário em alunos do $3^{\circ}$ ano do colégio Juan XXIII da Macaravita
\end{abstract}

Henry Alberto Macias Uribe

Magister en Pedagogía Docente Magisterio henrymacias821@gmail.com

\section{Resumen}

Este trabajo es el resultado de una investigación que se hizo con estudiantes del grado tercero de primaria del colegio Juan XXIII del municipio de Macaravita del departamento de Santander, Colombia. Consistió en indagar por cómo la planeación de los escritos a partir del propósito comunicativo, mensaje y destinatario, contribuyen a mejorar el proceso de escritura de los estudiantes, y en ese sentido llevar a desarrollar procesos de escritura de textos coherentes y cohesivos. En el aspecto metodológico se recurre al paradigma cualitativo y al diseño de investigación - acción.

Palabras clave: Escritura, planeación de los escritos, propósito comunicativo, mensaje, destinatario

\footnotetext{
${ }^{1}$ Artículo de investigación científica y tecnológica resultado de la investigación: La planeación de los escritos a partir del propósito comunicativo, mensaje y destinatario en estudiantes de grado 3 del colegio Juan XXIII de Macaravita. Caso: Colegio público-Urbano colegio Juan XXIII de Macaravita, Santander.
} 
La planeación de los escritos a partir del propósito comunicativo, mensaje y destinatario en estudiantes de grado 3 del colegio Juan XXIII de Macaravita

\begin{abstract}
This work is the result of a study done with third grade students of the Juan XXIII primary school in the municipality of Macaravita in the department of Santander, Colombia. It consisted of inquiring how the planning of the writings from the communicative purpose, message and recipient, contribute to improve the writing process of students; and in that sense, lead to develop processes of writing coherent and cohesive texts. In the methodological aspect, the qualitative paradigm and the research - action design are used.
\end{abstract}

Key words: Writing, writing planning, communicative purpose, message, recipient

\title{
Resumo
}

Este trabalho é o resultado de um estudo feito com alunos do terceiro ano da escola primária Juan XXIII no município de Macaravita, no departamento de Santander, Colômbia. Consistia em indagar como o planejamento dos escritos a partir do propósito comunicativo, mensagem e destinatário contribuiu para melhorar o processo de escrita dos alunos; e nesse sentido, levam a desenvolver processos de escrita de textos coerentes e coesos. No aspecto metodológico, utiliza - se o paradigma qualitativo eo desenho pesquisa - ação.

Palavras-chave: redação, redação do planejamento, finalidade comunicativa, mensagem, destinatário.

\section{Introducción}

El aula no es el único lugar en el que se presentan situaciones de enseñanza-aprendizaje, (Font, Badia, i Muntada, Muñoz, \& Cabaní, 1994), Sin embargo, sí es el sitio en el que confluye el conocimiento $y$ hay una sistematización que trae consigo un proceso estandarizado, es decir, hay un estudiante que aprende y un docente que enseña. Lo anterior es la descripción del escenario ideal de un aula, pero la realidad que deben enfrentar los docentes en nuestro país es muy diferente. En primaria es común encontrarse con niños que no saben ni leer ni escribir (Barboza \& Peña, 2014) y no solo en primaria, vale la pena acotar que incluso algunos llegan a la universidad sin las competencias básicas de lectura y escritura, tal como lo señalan las investigaciones de Carlino (2005).

Es por eso que con base en lo anterior se hizo una indagación 
por cómo la planeación de los escritos a partir del propósito comunicativo, mensaje y destinatario, contribuyen a mejorar el proceso de escritura en los estudiantes de tercero de primaria del colegio Juan XXIII del municipio de Macaravita, y en ese sentido desarrollar procesos de escritura y acompañar la creación de textos coherentes y cohesivos son finalmente los objetivos del presente trabajo.

La planeación de los escritos es de vital importancia, ya que es la que le permite al estudiante, por un lado generar las ideas que quiere comunicar $y$ por otro organizar en una estructura su texto para que el lector pueda hacer una lectura amena del texto, (Echeverri \& Romero, 1992). Obviamente la planeación de un escrito por un niño de primaria difiere de la de un estudiante de bachillerato, un tecnólogo, un universitario $\mathrm{o}$ un escritor profesional. Por eso en el aspecto de la planeación escrita en el contexto de la educación primaria, se tiene en cuenta el propósito comunicativo (qué quiero decir y para qué lo quiero decir), mensaje y destinatario (A quién se lo quiero comunicar y qué información tengo), acorde con lo estipulado por los derechos básicos de aprendizaje del ministerio de educación nacional Ministerio de Educación MEN (2015). Además en este proceso se tuvieron en cuenta aspectos básicos tanto de coherencia como de cohesión en los textos presentados por los niños acorde a su nivel de estudio.

En cuanto a la coherencia acorde con diferentes autores (Aznar, Anglés, Cros, \& Quintana, 1991; Gómez Veiga \& Vieiro Iglesias, 2000; Louwerse, 2004) se observa en los textos al identificar una idea central, la permanencia de esta a lo largo del texto (sin que se introduzcan ideas nuevas sin relación o digresiones) al significado de un texto en su totalidad y abarca las relaciones de las palabras con el contexto y al interior del texto. Se basa en la estabilidad y la consistencia temática asociada a la macroestructura (contenido) y a la superestructura (esquema de organización) del texto. $\mathrm{La}$ coherencia se construye a partir de la información dada y de las inferencias que activan los lectores para interpretar un texto a partir de conocimientos previos. En palabras de Anglés, E. A., Quintana, L., \& Cros, A. (1991). La coherencia textual y la lectura se entienden:

..."como nocontradictoriedad: ausencia de contradicción; en este sentido, un texto coherente sería, entre otras cosas, un texto sin contradicciones, sin ideas que no se relacionan con el tema. Ideas inconexas: ideas vagas, que no muestran 
La planeación de los escritos a partir del propósito comunicativo, mensaje y destinatario en estudiantes de grado 3 del colegio Juan XXIII de Macaravita

\section{claramente una relación semántica” (p.45).}

En cuanto al segundo aspecto, los elementos cohesivos de un texto están basados en elementos lingüísticos explícitos (es decir, palabras, rasgos, claves, señales, constituyentes) y sus combinaciones. El método generalizado para aumentar la cohesión textual consiste en aumentar, en el nivel superficial, los indicadores de relaciones entre las ideas del texto. Esas modificaciones pueden ir desde agregar información de bajo nivel, como sería la identificación de referentes anafóricos, términos sinónimos, enlaces conectores, o encabezados, hasta proporcionar información previa/general no expresada en el texto. Se puede facilitar y mejorar la comprensión textual cuando se reescriben los textos mal redactados, de modo que resulten más cohesivos y entreguen al lector toda la información que se requiere para una fácil comprensión(Louwerse, 2004).

\section{Metodología}

La metodología que se llevó a cabo en este trabajo tiene un enfoque cualitativo que se caracteriza por explorar los fenómenos en profundidad, se conduce en ambientes naturales y los significados se extraen de los datos y no se fundamenta en la estadística. Además es un proceso inductivo, en el que a partir de un caso particular de llega a conclusiones generales, recurrente y donde se analiza múltiples realidades subjetivas. Con relación a las bondades se tiene que la profundidad de significados, amplitud, riqueza interpretativa, además de que contextualiza el fenómeno y no tiene secuencia lineal (Sampieri, Collado, \& Lucio, 2014) para la investigación se seleccionó al grupo de estudiantes de grado tercero de primaria de una institución educativa pública del municipio de Macaravita, Santander. La elección se hizo por conveniencia debido a la accesibilidad de proximidad que como docente se tiene a los estudiantes, con la consideración de que la participación de ellos es voluntaria.

Además, se reitera que ésta investigación parte de la reflexión acerca del proceso de escritura de los estudiantes de tercero primaria para evidenciar los problemas que impiden el manejo de la misma.

El enfoque cualitativo consiste: "En la búsqueda cualitativa, en lugar de iniciar con una teoría y luego "voltear" al mundo empírico para confirmar si ésta es apoyada por los datos y resultados, el investigador comienza examinando los hechos en sí y en el proceso desarrolla una teoría coherente para representar lo que observa. Dicho de otra forma, las investigaciones cualitativas se 
basan más en una lógica y proceso inductivo (explorar y describir, y luego generar perspectivas teóricas). Van de lo particular a lo general. Por ejemplo, en un estudio cualitativo típico, el investigador entrevista a una persona, analiza los datos que obtuvo y saca conclusiones; posteriormente, entrevista a otra persona, analiza esta nueva información y revisa sus resultados y conclusiones; del mismo modo, efectúa y analiza más entrevistas para comprender el fenómeno que estudia. Es decir, procede caso por caso, dato por dato, hasta llegar a una perspectiva más general.(Sampieri et al., 2014)

En el presente trabajo se considera este enfoque porque se quiere llegar a la comprensión de un fenómeno social presente en la Institución Juan XXIII, en lo que refiere a la escritura en los niños, a partir del manejo de información para la elaboración de hallazgos ya que "Con él término "investigación cualitativa", se entiende cualquier tipo de investigación que produce hallazgos a los que no se llega por medio de procedimientos estadísticos u otros medios de cuantificación(Strauss, Corbin, \& Zimmerman, 2002). Como docentes de aula, se asume el rol de investigador por contar con acceso al ambiente natural donde se desarrolla la investigación con lo cual el fenómeno al ser estudiado será conocido e interpretado en el espacio y tiempo en el que se desarrolla cotidianamente, sin forzar ni provocar hechos o situaciones que rompan con esa naturalidad. Pues el contexto es parte de la información y el ambiente influye en el tipo de dato que se va a construir(Denzin \& Lincoln, 2012).

El diseño metodológico es Investigación-Acción (IA) ya que se considera pertinente para esta propuesta porque permite, en lo que refiere al problema, ayudar en la búsqueda de planes posibles y la formulación de soluciones (Mckernan, 1999, p. 28), de igual manera propicia el cambio social y como transformación de la realidad, las personas tienen un papel en ese proceso de cambio. En el contexto educativo permite observar situaciones de aula, y como docente investigador evidenciar cómo los estudiantes realizan su proceso de escritura, al respecto Sandín (2000) señala que:

La investigación-acción se encuentra ubicada en la metodología de investigación orientada a la práctica educativa. Desde esta perspectiva, la finalidad esencial de la investigación no es la acumulación de conocimientos sobre la enseñanza o la comprensión de la realidad educativa, sino, fundamentalmente, aportar información que guíe la toma de decisiones y los procesos de cambio para la mejora de la 
misma. Justamente, el objetivo prioritario de la investigaciónacción consiste en mejorar la práctica en vez de generar conocimientos; así, la producción y utilización del conocimiento se subordina a este objetivo fundamental y está condicionado por él (p.30).

De modo que mejorar las prácticas de escritura es una labor plausible desde la IA como fundamentación del cambio educativo, es por esto que al respecto Kemmis y McTaggart según (Paz, 2003. p.35) señalan que: "Significa darse cuenta de que las clases, las escuelas y la sociedad de hoy son resultados de un proceso de formación social e histórica y que, para lograr una forma diferente de clases, escuelas o sociedades, se debe emprender un proceso de reforma o transformación: una lucha por una reforma"

En el mismo sentido para Elliot(Elliott, 1990) la IA es:

... el estudio de una situación social para tratar de mejorar la calidad de la acción en la misma. Su objetivo consiste en proporcionar elementos que sirvan para facilitar el juicio práctico en situaciones concretas y la validez de las teorías e hipótesis que genera no depende tanto de pruebas "científicas" de verdad, sino de su utilidad para ayudar a las personas a actuar de modo más inteligente y acertado. En la investigación-acción, las "teorías" no se validan de forma independiente para aplicarlas luego a la práctica, sino a través de la práctica (p.36).

Siguiendo el planteamiento de Elliot, la IA posibilita la resolución de problemas en el aula en la medida que como docentes se esté en constante formación y capacitados para implementar nuevos enfoques que resulten innovadores dentro de un contexto de enseñanza aprendizaje, todo con el fin de transformar y mejorar no sólo la educación sino la sociedad misma. Por último, pese a que en rasgos generales la investigación acción se desarrolla siguiendo un modelo en espiral en ciclos sucesivos que incluyen diagnóstico, planificación, acción, observación y reflexión - evaluación y que el proceso de investigación acción es descrito con matizaciones diferentes según autores, variando en cuanto a su complejidad(Herreras, 2004), es de aclarar que para esta investigación se considera el modelo propuesto por Elliot donde se enfatiza dos importantes características de la IA, a saber, la teoría y la práctica.

En la presente investigación participaron 19 estudiantes de tercero primaria conformado por 9 niños y 10 niñas, con edades 
comprendidas entre los 8 hasta los 9 años.

Las técnicas e instrumentos fueron propios de la investigación cualitativa (Barba, 2013; Denzin \& Lincoln, 2012; Esteban, 2003; Paz, 2003; Strauss et al., 2002), así dentro de las técnicas se utilizó la entrevista estructurada, el grupo focal, taller investigativo $y$ observación participante, así mismo dentro de los instrumentos se usó la guía de entrevista, la guía de grupo focal, guía de taller ,diario de campo y grabación guía del grupo focal. Todo con el fin de dar una interpretación acorde con los objetivos del presente trabajo.

Para el análisis de datos se recurrió a la ayuda de la hoja de cálculo excel, el procesador de texto word $y$ finalmente el software Atlas ti, a través del cual se establecieron las categorías de análisis.

\section{Resultados}

La planificación y la revisión de los textos que hace el docente a modo de retroalimentación y a la vez estimulando la reescritura resulta en un fuerte aliado, porque a medida que el estudiante va construyendo su texto, este es reordenado y releído constantemente. Lo que a su vez le permite al estudiante reflexionar acerca de las ideas que para él son las más importantes y luego encontrar las palabras precisas que le permitan expresar la idea a través de la escritura en un párrafo con elementos básicos tanto de coherencia como de cohesión.

Sin embargo, en algunos estudiantes se presentó el caso de que escribir les resultaba un ejercicio complejo, tanto escribir las ideas como hacer una jerarquización entre estas para establecer cuáles eran las ideas más importantes frente a las otras era una tarea compleja, pero con la ayuda del docente se pudo trabajar en esos aspectos. Así mismo dentro de la planificación de la escritura el propósito comunicativo (qué quiero decir y para qué lo quiero decir) resultaba complejo porque los estudiantes no respondían con claridad a preguntas como qué quiero decir y para qué.

Las entrevistas, sin embargo, señalan que el asesoramiento constante del docente les genera la confianza suficiente para llevar a cabo su proceso de escritura dejando claro que uno de los proceso más complejos para ellos es empezar con el acto de escritura, pero que una vez tienen los elementos suficientes y claros, esto hace que la escritura avance con más fluidez.

Por último, y a modo de conclusión, se puede afirmar que los estudiantes de primaria de grado tercero, tienen el potencial de escribir un texto con jerarquización de ideas que se 
La planeación de los escritos a partir del propósito comunicativo, mensaje y destinatario en estudiantes de grado 3 del colegio Juan XXIII de Macaravita

expresan de manera coherente y con cohesión y que dicho proceso con la práctica puede ir mejorando cada día más, esto gracias a la planeación de los escritos a partir del propósito comunicativo, mensaje y destinatario que junto con la constante asesoría del docente resultan en un factor de apoyo importante en el ejercicio de escritura de los estudiantes, que sin embargo, consiste en un proceso minucioso que debe erigirse desde el currículo de la institución.

\section{Referencias}

Aznar, E., Anglés, E. A., Cros, A., \& Quintana, L. (1991). Coherencia textual y lectura (Vol. 6). HORSORI EDITORIAL, SL.

Barba, J. J. (2013). La investigación cualitativa en educación en los comienzos. Investigación cualitativa en educación musical, 298, 23.

Barboza, F. D., \& Peña, F. J. (2014). El problema de la enseñanza de la lectura en educación primaria. Educere, 18(59).

Benavides Cáceres, D. R., \& Sierra Villamil, G. M. (2013). Estrategias didácticas para fomentar la lectura crítica desde la perspectiva de la transversalidad. REICE. Revista Iberoamericana sobre Calidad, Eficacia y Cambio en
Educación, 11(3). Recuperado a partir

http://www.redalyc.org/resum en.oa?id=55128038004

Carlino, P. (2005). Escribir, leer y aprender en la universidad. Buenos Aires: Fondo de cultura económica.

Chaves, L. G., \& Romero, R. F. (2013). Desarrollo de habilidades de pensamiento inferencial y comprensión de lectura en niños de tres a seis años. PANORAMA, 5(9). Recuperado a partir de http://journal.poligran.edu.co/ index.hp/panorama/article/vie $\mathrm{w} / 39$

Denzin, N. K., \& Lincoln, Y. (2012). Manual de investigación cualitativa. Gedisa.

Echeverri, C. C., \& Romero, R. F. (1992). La escritura como proceso. Revista educación y cultura. Volumen, (28), 64-68. Recuperado a partir de http://media.utp.edu.co/refere nciasbibliograficas/uploads/re ferencias/articulo/660-laescritura-como-procesopdfaZPF0-articulo.pdf

Esteban, M. P. S. (2003a). Investigación cualitativa en educación: fundamentos $y$ tradiciones. McGraw-Hill.

Esteban, M. P. S. (2003b). Investigación cualitativa en educación: fundamentos $y$ 
tradiciones. McGraw-Hill.

Esteban, M. P. S. (2003c). Investigación cualitativa en educación: fundamentos y tradiciones. McGraw-Hill.

Font, C. M., Badia, M. C., i Muntada, M. C., Muñoz, M. P., \& Cabaní, M. L. P. (1994). Estrategias de enseñanza y aprendizaje: Formación del profesorado y aplicación en la escuela (Vol. 112). Grao.

Gómez Veiga, I., \& Vieiro Iglesias, P. (2000). Coherencia textual, conocimiento previo y comprensión. Recuperado a partir de http://ruc.udc.es/dspace/bitst ream/handle/2183/6752/RGP_ 6-113.pdf? sequence $=1$

Guerrero Rodríguez, D. A., \& others. (2011). Relación entre metacognición y composición de textos argumentativos. estudio a través de la implementación de una secuencia didáctica/Relationship between metacognition and composition argumentative texts. Study through the implementation of sequence learning. Universidad Nacional de Colombia. Recuperado a partir de http://www.bdigital.unal.edu.c o/4380/

Herreras, E. B. (2004). La docencia a través de la investigaciónacción. Revista Iberoamericana de Educación. Recuperado de http://www. rieoei. org/deloslectores/682Bausela. PDF. Recuperado a partir de http://www.academia.edu/do wnload/45393698/Bausela4.pdf

Linan-Thompson, S. (2013). La importancia del desarrollo de lectoescritura: de la cuna a la escuela.

Lineamientos curriculares Ministerio de Educación Nacional de Colombia. (s/f). Recuperado el 21 de agosto de 2017, a partir de http://www.mineducacion.gov. co/1759/w3-article339975.html

Louwerse, M. M. (2004). Un modelo conciso de cohesión en el texto y coherencia en la comprensión. Revista signos, 37(56), 41-58. Recuperado a partir de http://www.scielo.cl/scielo.ph p?pid=S0718$09342004005600004 \&$ script $=\mathrm{s}$ ci_arttext\&tlng=en

McKernan, J. (1999). Investigaciónacción y currículum: métodos y recursos para profesionales reflexivos. Ediciones Morata.

McNamara, D. S. (2004a). Aprender del texto: Efectos de la estructura textual y las estrategias del lector. Revista signos, 37(55), 19-30. Recuperado a partir de 
http://www.scielo.cl/scielo.ph p?pid=S071809342004005500 002\&script=sci_arttext\&tlng=e $\mathrm{n}$

McNamara, D. S. (2004b). Aprender del texto: Efectos de la estructura textual y las estrategias del lector. Revista signos, 37(55), 19-30. Recuperado a partir de http://www.scielo.cl/scielo.ph p?pid=S071809342004005500 002\&script=sci_arttext\&tlng=e $\mathrm{n}$

Monereo Font, C., \& Badia Garganté, A. (2013). Aprendizaje estratégico y tecnologías de la información y la comunicación: una revisión crítica. Teoría de la Educación. Educación y Cultura en la Sociedad de la Información, 14(2). Recuperado a partir de http://www.redalyc.org/html/ 2010/201028055002/

Paz, M. (2003). Investigación cualitativa en educación. Fundamentos y tradiciones. Madrid. Mc Graw and Hill Interamericana de España.

Sampieri, R. H., Collado, C. F., Lucio, P. B., \& Pérez, M. de la L. C. (1998). Metodología de la investigación (Vol. 1). Mcgrawhill México. técnicas y procedimientos para desarrollar la teoría fundamentada. Universidad de Antioquia Medellín.

Valderrama Araque, Y. T., Barragán Ramírez, C., \& Maya Poveda, C. A. (2017). Estrategias pedagógicas $\mathrm{y}$ didácticas para el fortalecimiento de procesos metacognitivos para la comprensión lectora en estudiantes del grado segundo del Centro Educativo Rural Media Luna, anexo el Placer, corregimiento de Santa Elena. Recuperado a partir de http://ayura.udea.edu.co:8080 /jspui/handle/123456789/250 6

Strauss, A. L., Corbin, J., \& Zimmerman, E. (2002). Bases de la investigación cualitativa: 

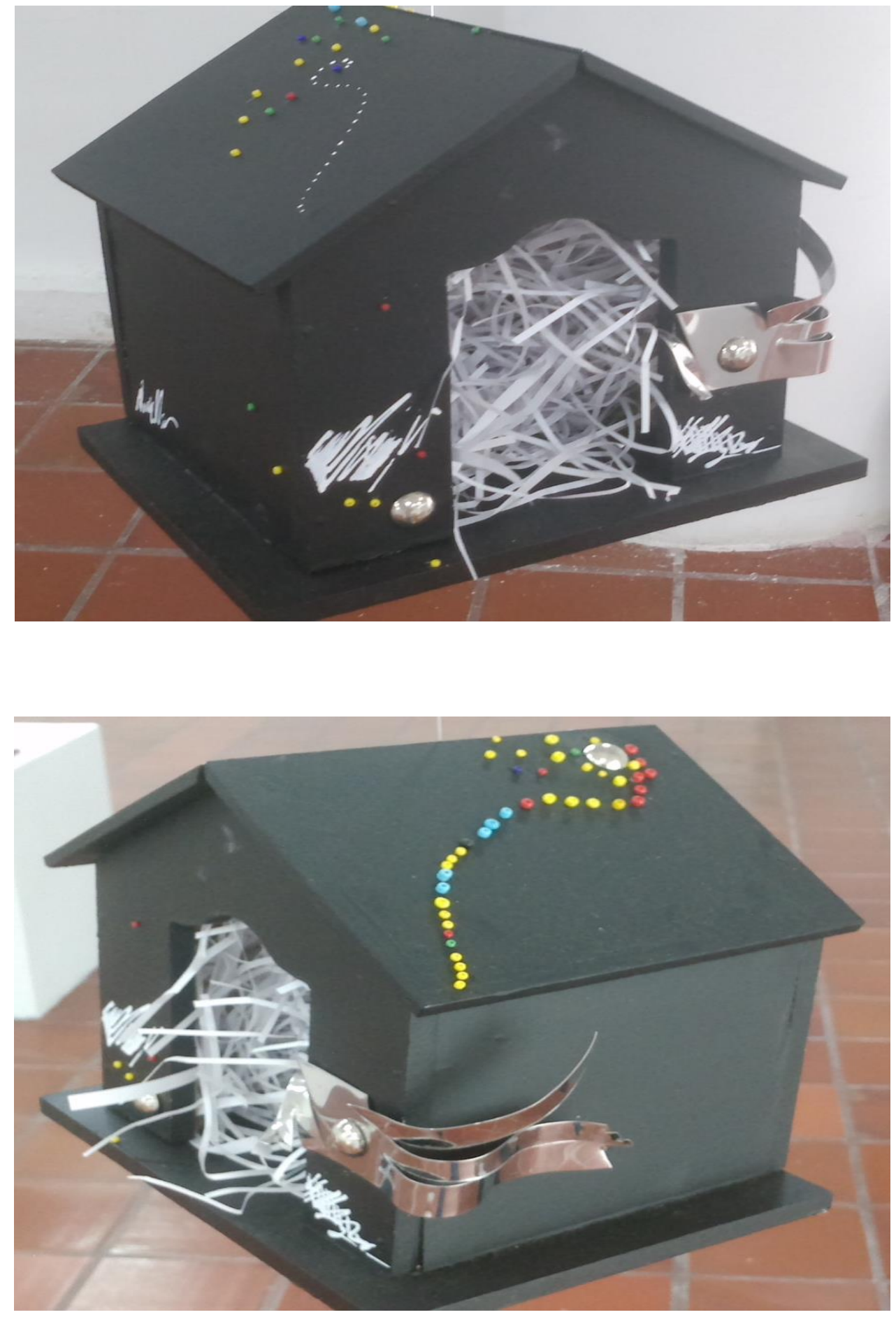

Obra: Desplumada y desplomada Autor: Javier Chinchilla N. Técnica: Mixta Territorio: Neiva - Pitalito 